\title{
Effect of Low-temperature Induction on Heat Mining Performance of EGS in Hot Dry Rocks
}

\author{
Wei Zhang, Zhan-qing Qu, and Tian-kui Guo
}

\begin{abstract}
As high temperature in hot dry rocks(HDR), the process of injecting cold fracturing fluid to form fractures and circulating working fluid to extract heat energy from enhanced geothermal system(EGS) all inevitably involve the low-temperature induction imposing on hot dry rocks. Considering the temperature-dependent parameter changes of matrix and fluid, we establish thermal-hydraulic-mechanical full coupling model to investigate the effect of low-temperature induction on heat mining performance of EGS. This paper begins with experimentally study to obtain the possible distribution of sub-fractures in EGS caused by the thermal stress cracking induced by thermal drawdown; Then we discuss the heat mining performance in terms of whether the sub-fractures are considered and whether the thermal stress aroused by low-temperature fluid circulation is taken account during mining process. We find that thermal stress induced by low-temperature circulation brings contraction of matrix and influences seepage capacity of fractures by changing effective stress in EGS mining.
\end{abstract}

Index Terms - Low-temperature induction, heat mining performance, thermal-hydraulic-mechanical(THM) coupling, hot dry rocks(HDR)

\section{INTRODUCTION}

Geothermal energy has attracted worldwide attention due to its stableness, cleanness, sustainability and large abundance of storage. HDR was deemed as one of the most promising new energy resources because of its high temperature $\left(150-650^{\circ} \mathrm{C}\right)$ and rich in resources [1]. However, the HDR matrix is impervious as it is located within subsurface of $3-10 \mathrm{~km}$ depths, making it tough to mine the high temperature heat energy by conventional hydrothermal exploitation method. Therefore, fracturing techniques are used to create artificial fractures or to improve the existing cracks in order to form the enhanced geothermal system(EGS), then the working fluids can be circulated in EGS to extract heat effectively.

The process of injecting low-temperature fracturing fluid to form fractures and circulating the working fluid to extract heat energy are all related to the variation of reservoir stress induced by thermal drawdown because of high temperature in

Manuscript received October 21, 2017; revised May 1, 2018. This work was supported the Fundamental Research Funds for the Central Universities (Grant No. 17CX06008); the Fundamental Research Funds for the Central Universities (Grant No. 17CX02077); the Applied Basic Research Project for Qingdao (Grant No. 17-1-1-20-jch).

Wei Zhang is with the China University of Petroleum, Upc, CO 266580 China (e-mail: 18562029717@163.com).

Zhan-qing Qu and Tian-kui Guo is with the China University of Petroleum, Upc, CO 266580 China (e-mail: quzhq@upc.edu.cn, guotiankui@126.com).
HDR. Fracture morphologies are affected by thermal stress cracking induced by thermal drawdown during the formation of EGS, then the variation of fracture morphology will influence the heat mining performance. Previous studies have shown that low-temperature induction is more conducive to the formation of fracture networks. Tomac.I et al (2014) certified the temperature contrast between the reservoir and injection fluids played an important role in the fracture initiation and propagation in EGS [2]. Julio Garcia et al (2015) and Jonny Rutqvist et al (2015) adopted micro seismic and pressure monitoring techniques in Geysers, which proved that the cooling thermal shock, injection pressure and the reopen of cracks existing initially created the wide range of fracture networks [3]. Guo LL (2016) used the ambient $\left(20{ }^{\circ} \mathrm{C}\right)$ temperature fracturing fluid to fracture the heated rock $\left(90^{\circ} \mathrm{C}\right)$, several fractures are formed [4].

Fractures are main flow path and heat transfer area after formation of EGS. Many scholars have launched related Multi-physical coupling research on heat mining of EGS. Yangsheng Zhao et al (2015) studied the evolution of reservoir temperature, fracture aperture, flow impedance and permeability in Teng Chong geothermal field by a THM coupling model [5]. Wieslaw Bujakowski et al (2015) considered that the volume and permeability of the fractured area have significant influence on the development of EGS by using TOUGH2 and a sedimentary thermal reservoir in central Poland was taken as an example [6]. Yan-Jun Zhang et al (2015) proved that it's possible to improve the economic efficiency of geothermal by staged fracturing of horizontal wells [7]. Zhan-qing Qu et al (2017) simulated the influence of possible artificial fracture morphology on heat mining performance of EGS [8]. However, the above studies have neglected the stress changes of matrix caused by thermal stress. Though researches by S. N [9] and Guo [10]. have taken thermal stress into account, they ignored the diversity of fractures in EGS. Zhi-xue Sun et al (2017) simulated the 2D fracture network in EGS, but they only studied the output temperature for different model parameters [11].

Therefore, it's necessary to carry out development characteristics research considering thermal stress during heat mining based on the fracture morphology fractured by low-temperature induced cracking, which have comprehensive indicators including production temperature, mass flow rate, heat mining rate and the alteration of fracture permeability. 


\section{INTERACTIONS OF THM COUPLING}

\section{A. Basic Assumptions}

(1) The flow seepage of fluid both in matrix and fractures obeys the Darcy`s law; (2) No chemical reactions occur between fluids and rocks; (3) The heat transfer accords with the Fourier`s law of heat conduction without taking the effects of thermal radiation into account; (4) There is no phase transformation because the reservoir temperature is below the boiling point of water; (5) The matrix is considered to be thermal elastic based on small strain assumption; (6) We consider the porous media to be isotropic.

\section{B. Governing Equations}

Based on above assumptions, the governing equations of THM coupling model considering low-temperature induction in EGS development can be expressed as follows:

Mass conservation equation

$$
\rho S \frac{\partial \mathrm{p}}{\partial t}+\nabla \cdot(\rho v)=Q_{m}
$$

The flow of liquid in the porous elastic medium of matrix is depicted by the mass balance equation in $2-1$. Where, $\rho$ is the matrix density, $\mathrm{kg} / \mathrm{m}^{3} ; \mathrm{S}$ is the storage coefficient of matrix rock; $t$ is the develop time; $\mathrm{Q}_{\mathrm{m}}$ is the term of seepage source, $\mathrm{kg} /\left(\mathrm{m}^{3} \cdot \mathrm{s}\right) ; \mathrm{v}$ is water flow velocity in matrix rock, $\mathrm{m} / \mathrm{s}$; The liquid flow in matrix follows Darcy`s law:

$$
\begin{gathered}
v=-\frac{k}{\mu} \nabla \cdot p \\
d_{f} \rho_{f} S_{f} \frac{\partial p}{\partial t}+\nabla_{\tau} \cdot\left(d_{f} \rho_{f} v_{f}\right)=Q_{f} \\
v_{f}=-\frac{k_{f}}{\mu} \nabla_{\tau} \cdot p
\end{gathered}
$$

The mass balance equation in 2-3 describes the flow of liquid in the porous elastic medium of discrete fractures. Where, $d_{f}$ is the fracture thickness, $\mathrm{m} ; \rho_{\mathrm{f}}$ is the fracture density, $\mathrm{kg} / \mathrm{m}^{3} ; \mathrm{S}_{\mathrm{f}}$ is the storage coefficient in fracture; $\mathrm{Q}_{\mathrm{f}}$ is the flow exchange between matrix and fractures; $\mathrm{k}_{\mathrm{f}}$ is the fracture permeability, $\mathrm{m}^{2} ; \nabla \tau$ is the tangential gradient along the fracture.

Energy balance equation

$$
\begin{gathered}
\left(\rho C_{p}\right)_{e f f} \frac{\partial T}{\partial t}+\rho_{w} C_{p w} v \cdot \nabla T+\nabla \cdot q=\Phi_{T} \\
d_{f}\left(\rho C_{p}\right)_{e f f} \frac{\partial T}{\partial t}+d_{f} \rho_{w} C_{p w} v \cdot \nabla T+\nabla \cdot q_{f}=Q
\end{gathered}
$$

The equation 2-5 describes the heat transfer in porous matrix, where, $\left(\rho \mathrm{C}_{\mathrm{p}}\right)_{\mathrm{eff}}$ is the effective heat capacity obtained by volume average, $\left(\rho C_{\mathrm{p}}\right)_{e f f}=\rho_{s} C_{p s}(1-\phi)+\rho_{w} C_{p w} \phi$; $\mathrm{C}$ is the specific heat capacity; $q=-k_{\text {eff }} \nabla T$ and $\mathrm{K}_{\mathrm{eff}}$ is the effective thermal conductivity obtained by volume average, $k_{\text {eff }}=\lambda_{s}(1-\phi)+\lambda_{w} \phi ; v$ is the fluid velocity in matrix, when the fluid obeys Darcy`s law, it satisfies equation 2-2; $\Phi_{T}$ is the term of heat source, $\mathrm{J} /\left(\mathrm{m}^{3} \cdot \mathrm{s}\right)$. The subscript $\mathrm{s}$ and $\mathrm{w}$ respectively corresponding matrix and water; $\varphi$ is matrix porosity.

The equation 2-6 describes energy exchanges between matrix and fluid in fractures, temperature of matrix and fluid are different on the fracture surface, so heat transfer between rock matrix and fluid occurs in fractures. Q is the energy that the flowing fluid in fractures absorbs from matrix. It is assumed that the heat transfer between flowing fluid and matrix obeys the Newton`s heat transfer law, the heat transfers from matrix to flowing fluid is depicted in equation 2-7.

$$
Q=h\left(T_{s}-T_{f}\right)
$$

Mechanical field equation

$$
G u_{i, j j}+\frac{G}{1-2 v} u_{j, j i}-\alpha_{B} p_{, i}-K^{\prime} \alpha_{T} T_{, i}+F_{i}=0
$$

where, $\mathrm{u}_{\mathrm{i}}$ is displacement component; $\mathrm{G}, v$ is the Shear modulus and Poisson ratio, $G=E / 2(1+v)$; E is Young`s modulus; $F_{i}$ is the body force per unit volume in the i-direction; $-\alpha_{B} p_{, i}$ presents the action of water pressure(seepage force under porous pressure), where $\alpha_{B}$ is Biot coefficient; $-K^{\prime} \alpha_{T} T_{, i}$ denotes the function of thermal stress, where $\alpha_{T}$ is matrix thermal expansion coefficient, $K^{\prime}$ is the Bulk Elastic Modulus, $K^{\prime}=E /(1-2 v)$.

In order to represent the fracture stiffness, the internal boundary of fractures is described in a weak form [5]. The fractures are approximately depicted by a pair of normal and shear displacement surfaces; the deformation equation of matrix as following:

$$
\begin{aligned}
& u_{n}=\sigma_{n}^{\prime} / k_{n}, u_{s}=\sigma_{s}^{\prime} / k_{s} \\
& \sigma_{n}^{\prime}=\sigma_{n}-\alpha_{B} p, \sigma_{s}^{\prime}=\sigma_{s}
\end{aligned}
$$

where, $u, \sigma, \sigma^{\prime}, k$ respectively indicate the displacement, total stress, effective stress and stiffness, the subscript $\mathrm{n}$ and $\mathrm{s}$ denote the normal and shear directions of fracture surface.

\section{Interaction of THM Coupling}

(1) Influence of seepage field on temperature field: with the flowing of cold fluid, heat exchange between fluid and hot matrix causes the change of balanced temperature filed;

(2) Influence of temperature field on seepage field: the physical characteristics of fluid flowing both in matrix and fractures are no longer constant, and change with temperature due to high temperature and high pressure in HDR [8].

(3) Influence of temperature field on stress field: the change of temperature affects the inherent physical and mechanical properties of matrix; besides, thermal stress created by temperature change lead to the alteration of original stress field. The young's modulus at different temperature obtained by experiment [12]. 
(4) Influence of stress field on temperature field: heat will be generated during deformation caused by stress field variation, meanwhile the thermal conductivity will be affected by matrix deformation.

(5) Influence of seepage field on deformation field: the effective stress will be impacted by porous pressure evoked by fluid injection.

(6) Influence of stress field on seepage field: the change of stress filed leads to the contraction and expansion of matrix porous and the aperture of the fractures, so the permeability of matrix and fractures changes.

The permeability of matrix is extremely low against with fractures, so the variation of matrix permeability is neglected.

$$
K_{f}=K_{0} \exp \left(-\alpha \sigma_{n}^{\prime}\right)
$$

where, $K_{f}$ is fracture permeability, $K_{0}$ is the initial value of fracture permeability, $\sigma_{n}^{\prime}$ denotes the effective stress imposing on fracture surface, $\alpha$ is a normalized constant related to the deformation of fractures.

\section{MODEL DESCRIPTION AND CALCULATION CONDITIONS}

\section{A. Hot Rock Cracked Experiment under the Low-temperature Induction Conditions}

The existence of thermal stress cracking induced by injection of low-temperature fracturing fluid and its important role in the EGS heat mining have been confirmed by many scholars. However, the possible formation of fracture morphologies remains unclear. In order to conclude the distribution of sub-fractures around the main fracture due to the thermal stress cracking, hot core specimens are used to conduct the experiment of thermal stress cracking induced by low-temperature. Specific schemes are as follows: (1) the core with $25 \mathrm{~mm}$ diameter was sliced and observed under microscope to observe if there were any cracks on the surface; (2) then heat the specimens at $200^{\circ} \mathrm{C}$ with $6 \mathrm{~h}$ (the drying box is heated by air convection, so the cracks created by sharply heated is ignored) (3) take out the heated core specimen $\left(200^{\circ} \mathrm{C}\right)$ then make sure it contacts the normal temperature water $\left(20^{\circ} \mathrm{C}\right)$ immediately; (4) after the interaction of hot core and low temperature water we use microscope observe if there are any cracks on surface. This procedure is considered as one time of thermal stress cracking induced by thermal drawdown. We observe the development of fracture morphology by duplicate the cracking experiment (repeat 1-4 steps).

Repeated cracking experiment was conducted by using the core buried $3650 \mathrm{~m}$ depth in Jiangsu oilfield (the density is $2.56 \mathrm{Kg} / \mathrm{m}^{3}$ and the compressive strength is $176 \mathrm{MPa}$ under the confining pressure $30 \mathrm{MPa}$ ). The 1 st thermal stress cracking induced by thermal drawdown emerges the narrow aperture fracture(stage1) as shown in Fig1. Then we repeated the cracking procedure (2nd time), the aperture degree of stage 1 fracture increases and the stage 2 fracture appears in the vicinity of large particles, which aperture is narrower than stage 1 fracture. The stage 3 fracture emerges after 3rd time thermal cracking as expected. Therefore, the fracture morphology is related to the times of cooling thermal cracking, and the difference in fracture permeability at different fracture stages cannot be neglected.
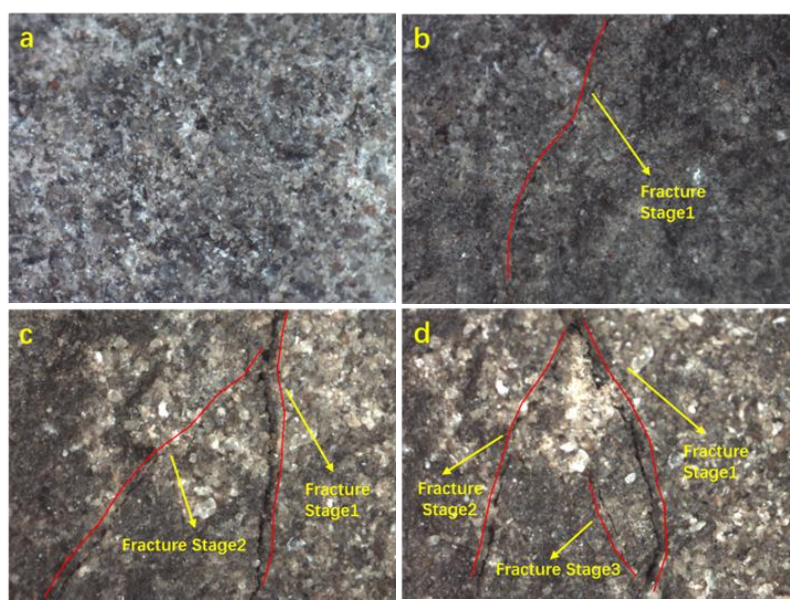

Fig. 1. Experimental results of thermal stress cracking induced by low-temperature under microscope ( $a$, initial state; $b$, after 1 st thermal stress cracking; c, after 2nd time cracking; d, after 3rd time cracking).

\section{B. Model Description}

Currently, studies of THM coupling in EGS heat mining hardly consider the function of thermal stress cracking induced by injection of low-temperature fracturing fluid, while it`s impractical to adopt a single fracture model for EGS development simulation. Meanwhile, the spacing between injection and production is the key factor that determines the duration of EGS, which needs more than $300 \mathrm{~m}$ in order to guarantee the duration of heat mining long enough. However, hydraulic fracturing can only make fractures $150-200 \mathrm{~m}$ at present, which requires the fracturing stimulation in both injection and production wells separately. As the important flow channel in EGS, connectivity of artificial fractures is critical. Accordingly, a 2D description of sub-fractures around the main fracture created by thermal drawdown during the injection of low-temperature fracturing fluid in a 300 $\mathrm{m} \times 300 \mathrm{~m}$ thermal reservoir is depicted based on experiment, as shown in Fig2(b). Then this model will be compared with the single fracture model(Fig2(a)) for heat mining performance.

\section{Calculation Conditions}

The above model is used to simulate the whole heat extraction process in EGS. THM coupling analysis starts with the water injection, each time step is $1 \mathrm{~d}$ and the total simulation time is 40 years. The initial and boundary conditions are as follows: (1) Seepage field: To ensure the fluid circulation in system, a given pressure boundary is adopted. Assuming the injection well gives a pressure of $37 \mathrm{MPa}$ and the production well gives a pressure of $30 \mathrm{MPa}$, the other conditions are impermeable boundaries. The well diameter is $0.2 \mathrm{~m}$. Due to almost no fluid exists in the hot dry rock the initial pressure in reservoir is considered 33MPa. (2) Temperature field: The injection temperature is $20^{\circ} \mathrm{C}$. Model boundaries are set as open boundaries to simulate the heat transfer between the surrounds matrix and EGS. For matrix and water in reservoir, the initial temperature is both $200^{\circ} \mathrm{C}$. (3) Stress field: The in-situ stress in y-direction is $40 \mathrm{MPa}$ and in $\mathrm{x}$-direction is $55 \mathrm{MPa}$, the other conditions are fixed 
boundaries. Besides, the research emphasizes on the influence of pressurized fluid injection and thermal stress evoked by exploitation on stress field and their coupling.
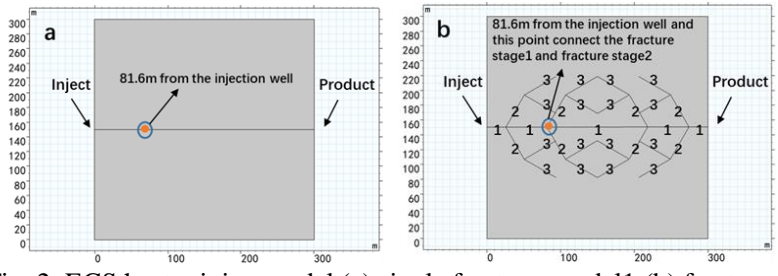

Fig. 2. EGS heat mining model (a) single fracture, model1; (b) fracture network considering the sub-fractures around main fracture caused by thermal stress cracking, model2; the permeability probes are also shown in a and $\mathrm{b}$.

In this work, the full coupling of solid deformation, fluid seepage and heat transfer is realized by COMSOL Multiphysics, and the 2D models are solved. Specifically, the mesh of single fracture(model1) is divided into 642 domain elements and 78 boundary elements; the mesh of fracture network(model2) is divided into 1946 domain elements and 248 boundary elements, model parameters are shown in TABLE I.

TABLE I: MODEL PARAMETERS

\begin{tabular}{cccc}
\hline \hline$\rho_{\mathrm{m}} /\left(\mathrm{kg} / \mathrm{m}^{3}\right)$ & $\mathrm{k}_{\mathrm{m}} /\left(\mathrm{m}^{2}\right)$ & $\lambda_{\mathrm{m}} /(\mathrm{W} / \mathrm{m} / \mathrm{K})$ & $\mathrm{E}_{0} /(\mathrm{GPa})$ \\
2700 & $1 \times 10^{-17}$ & 3.1 & 40 \\
\hline $\mathrm{S} /(1 / \mathrm{Pa})$ & $\alpha_{\mathrm{B}}$ & $\alpha_{\mathrm{T}} /(1 / \mathrm{K})$ & $\rho_{\mathrm{f}} /\left(\mathrm{kg} / \mathrm{m}^{3}\right)$ \\
$1 \times 10^{-8}$ & 1 & $3.6 \times 10^{-6}$ & 1200 \\
\hline $\mathrm{d}_{\mathrm{f} 1} /(\mathrm{m})$ & $\mathrm{d}_{\mathrm{f} 2} /(\mathrm{m})$ & $\mathrm{d}_{\mathrm{f} 3} /(\mathrm{m})$ & $\mathrm{S}_{\mathrm{f}} /(1 / \mathrm{Pa})$ \\
0.004 & 0.002 & 0.001 & $1 \times 10^{-9}$ \\
\hline $\mathrm{K}_{\mathrm{f} 1} /\left(\mathrm{m}^{2}\right)$ & $\mathrm{K}_{\mathrm{f} 2} /\left(\mathrm{m}^{2}\right)$ & $\mathrm{K}_{\mathrm{f} 3} /\left(\mathrm{m}^{2}\right)$ & $v$ \\
$5 \times 10^{-11}$ & $3 \times 10^{-11}$ & $2 \times 10^{-11}$ & 0.25 \\
\hline$\alpha /(1 / \mathrm{Pa})$ & $\mathrm{C}_{\mathrm{m}} /(\mathrm{J} / \mathrm{kg} / \mathrm{K})$ & $\mathrm{k}_{\mathrm{n}} /(\mathrm{GPa} / \mathrm{m})$ & $\mathrm{K}_{\mathrm{s}} /(\mathrm{GPa} / \mathrm{m})$ \\
$0.1 \times 10^{-6}$ & 950 & 100 & 30 \\
\hline \hline
\end{tabular}

\section{RESUlTS AND DisCUSSION}

In order to comparatively analyze the heat mining performance of EGS, we set permeability probes on the fractures as shown in Fig2, which describe the evolution of fracture permeability. In addition, the production mass flow rate, production temperature and heat mining rate are also used as evaluating indicators in this work [7], [8].

\section{A. Influence of Thermal Stress Cracking During EGS Formation}

In this part, we investigate the exploitation difference of M2 and M1 due to different flow channels, only considering difference of fracture morphology caused by thermal stress cracking in the formation of EGS without regard for the thermal stress imposing on matrix during heat mining and temperature-dependent parameters. Output mass flow rate(Fig3b) in M2 increased by nearly 2 times compared with M1 due to both the communication of sub-fractures and the flowing pressure induced by difference of permeability(Fig3d). Compared with single fracture, increasing of flow path enlarges the heat transfer area of working fluid, which elevates the temperature of output liquid(Fig3a). While at later development stage the temperature reduction scope in matrix around the fractures in M2 is larger than M1, as shown in Fig4, resulting in production temperature in M2 lower than M1 after 33-year development. The formation of sub-fractures increases the heat mining rate by $3 \mathrm{MW}(\mathrm{Fig} 3 \mathrm{c})$.

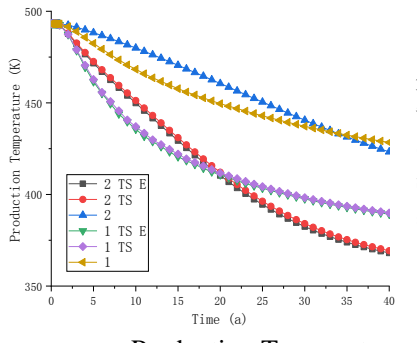

a Production Temperature

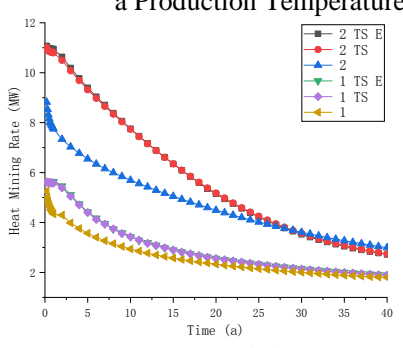

c Heat Mining Rate

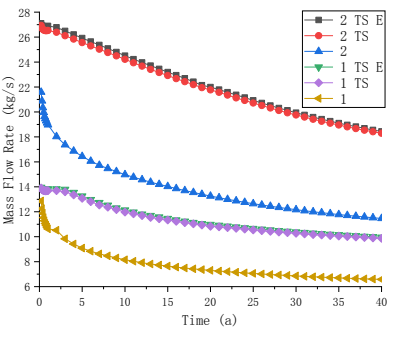

b Mass Flow Rate

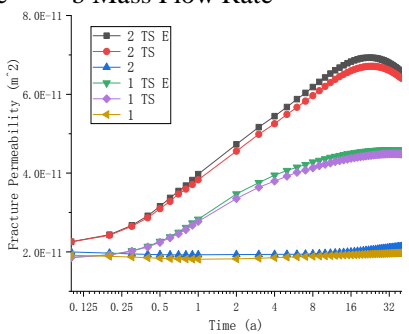

d Fracture Permeability
Fig. 3. Heat mining performance of EGS ' 2 ' denotes the fracture network model considering thermal stress cracking (Fig2b); ' 1 ' denotes the single fracture model formed under the conventional fracturing(Fig2a); 'TS' represents the thermal stress aroused by low-temperature fluid circulation during EGS exploitation; 'E' represents the influence of temperature on Young`s modulus is taken into account (position of permeability probes in Fig. 3d are shown in Fig. 2);
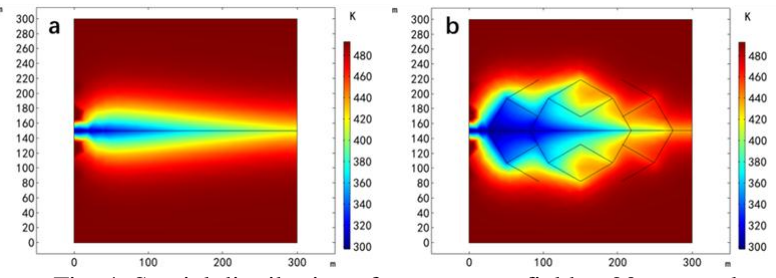

Fig. 4. Spatial distribution of temperature field at 20-year only considering difference of fracture morphology caused by thermal stress cracking induced by low-temperature fracturing fluids a. M1; b. M2

\section{B. Influence of Thermal Stress Imposing on Matrix During Heat Mining}

During heat mining, thermal stress induced by low-temperature fluid circulation changes the effective stress field, as shown in Fig5. The matrix around the fracture is still compressed when only under the action of water flowing pressure due to the presence of in-situ stress(Fig5a); while when the thermal stress is taken into account during water circulation, the stress field changes as the cooling of matrix(Fig5b). Variation of stress field creates the displacement of matrix(Fig6), fracture permeability advances under the combined function of water flowing pressure. As seen in Fig3d, the fracture permeability improves enormously against that neglecting the thermal stress both in M1 and M2, but the increment is different. The fracture permeability increase about 2 times at 1 -year in M2, reaches $40 \times 10^{-12} \mathrm{~m}^{2}$ and increase about 3times at 10 -year, reaching $62 \times 10^{-12} \mathrm{~m}^{2}$, but in M1 the fracture permeability only increase 2 times at 10a. It can be interpreted that the cooling scope of matrix is enlarged by the influence of fluid flowing through sub-fracture networks. The amplifying of cooling contraction range in matrix (Fig. 6) leads to the more dramatically 
improvement of fracture permeability.

With the increase of fracture permeability caused by thermal stress during water circulation in HDR, the output mass flow rate raises from $8 \mathrm{~kg} / \mathrm{s}$ to $12 \mathrm{~kg} / \mathrm{s}$ in $\mathrm{M} 1$ and from $15 \mathrm{~kg} / \mathrm{s}$ to $24 \mathrm{~kg} / \mathrm{s}$ in M2 (Fig $3 \mathrm{~b}$ ). However, the increase of fracture permeability and mass flow rate makes heat transfer in fracture insufficiently, resulting in the production temperature decrease(Fig3a). In particular, this mechanism is more pronounced in $\mathrm{M} 2$, which explains the faster temperature drawdown in M2. At early stage of development, due to the enormously raised mass flow rate, the heat mining rate grow dramatically; but with decrease of production temperature the increment of heat mining rate drawdown.

\section{Influence of the Temperature-dependent Young `s Modulus}

The young`s modulus at different temperature obtained by experiment [12]. The high reservoir temperature results in the reduction of Young`s modulus. Under the same temperature difference between matrix and injection water the effective stress imposing on the matrix surface raises due to the reduction of Young`s modulus (causing by thermal stress), which making the matrix deformation more serious and increasing the fracture permeability(Fig3d); meanwhile the small increment leads to a slight increase in output mass flow rate(Fig. 3b) and a slight decrease in output temperature (Fig. 3a).
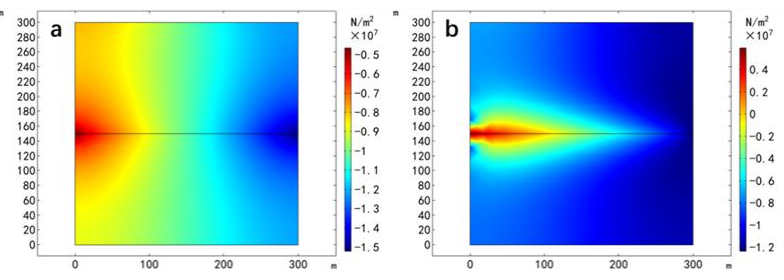

Fig. 5. Spatial distribution of stress field at 10-year in M1 a. without considering thermal stress aroused by low-temperature circulation $b$. considering thermal stress aroused by low-temperature circulation

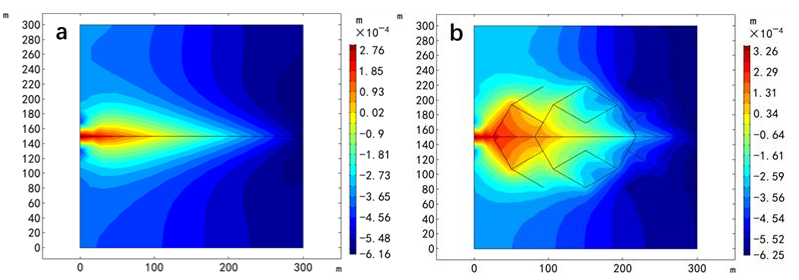

Fig. 6, Spatial distribution of displacement at 10-year a.M1; b. M2.

\section{CONCLUSIONS}

Considering the temperature-dependent parameter changes of rock and fluid, a full coupling of solid deformation, fluid seepage and heat transfer is established; then the $2 \mathrm{D}$ model is solved by COMSOL Multiphysics in order to clarify the influence of low-temperature induction on heat mining performance and its mechanism.

(1) Experiments prove that thermal stress cracking induced by thermal drawdown is beneficial to the construction of fracture networks during the formation of EGS, besides, the times of cooling treatment is critical to the fracture morphology.

(2) Fracture morphology difference caused by low-temperature fracturing during formation of EGS lead to the change of flow channels and alteration of thermal transfer area; Matrix contraction caused by thermal stress evoked by water circulation during heat mining transforms effective stress field, and promote the fracture permeability further; The stress field is also affected by temperature-dependent rock parameters.

(3) Through the above research, existence of sub-fractures plays an important role in EGS heat mining. Especially, high temperature environment in HDR is beneficial for the formation of sub-fractures caused by thermal stress cracking induced by low-temperature injection. So the accurate description of fracture morphology in EGS will be of significance in future research.

\section{ACKNOWLEDGMENT}

This work was jointly supported by the Fundamental Research Funds for the Central Universities (Grant No. 17CX06008); the Fundamental Research Funds for the Central Universities (Grant No. 17CX02077); the Applied Basic Research Project for Qingdao (Grant No. 17-1-1-20-jch).

\section{REFERENCES}

[1] C. R. Chamorro, J. L. García-Cuesta, and M. E. Mondéjar, "Enhanced geothermal systems in Europe: An estimation and comparison of the technical and sustainable potentials," Energy, 2014, vol. 65, pp. 250-263.

[2] I. Tomac and M. Gutierrez, "Micro-mechanics of hydro-thermo-mechanical fracture propagation in granite," ARMA, pp. 14-7148, 2014.

[3] J. Garcia, C. Hartline, M. Walters, M. Wright, J. Rutqvist, P. F.Dobson, and P. Jeanne, "The northwest geysers EGS demonstration project," California Part1: Characterization and Reservoir Response to Injection, 2015, vol. 63, pp. 97-119.

[4] L. L. Guo, "Test and model research of hydraulic fracturing and reservoir damage evolution in enhanced geothermal system," Ji Lin University, 2016.

[5] Y. S. Zhao, Z. J. Feng, Z. C. Feng, D. Yang, and W. G. Liang, "THM (Thermo-hydro-mechanical) coupled mathematical model of fractured media and numerical simulation of a 3D enhanced geothermal system at 573K and buried depth 6000-7000M," Energy, 2015.

[6] W. Bujakowski, A. Barbacki, M. Miecznik, and L. Pajak, "Modeling geothermal and operating parameters of EGS installations in the lower Triassic sedimentary formations of the central Poland area," Renewable Energy, 2015.

[7] Y. J. Zhang, L. L. Guo, Z. W. Li, Z. W. Yu, T. F. Xu, and C. Y. Lan, "Electricity generation and heating potential from enhanced geothermal system in Songliao Basin, China: Different reservoir stimulation strategies for tight rock and naturally fractured formations," Energy, 2015.

[8] Z. Q. Qu, W. Zhang, and T. K. Guo, "Influence of different fracture morphology on heat mining performance of enhanced geothermal systems based on COMSOL," International Journal of Hydrogen Energy, 2017.

[9] S. N. Pandey, A. Chaudhuri, and S. Kelkar, "A coupled thermo-hydro-mechanical modeling of fracture aperture alteration and reservoir deformation during heat extraction from a geothermal reservoir," Geothermics, 2017.

[10] B. Guo, P. C. Fu, Y. Hao, C. A. Peters, and C. R. Carrigan, "Thermal drawdown-induced flow channeling in a single fracture in EGS," Geothermics, 2016.

[11] Z. X. Sun, X. Zhang, Y. Xu, J. Yao, H. X. Wang, S. H. Lv et al., "Numerical simulation of the heat extraction in EGS with thermal-hydraulic-mechanical coupling method based on discrete fractures model," Energy, 2017.

[12] W. C. Zhu, J. Wei, J. Zhao, and L. L. Niu, "2D numerical simulation on excavation damaged zone induced by dynamic stress redistribution," Tunnelling and Underground Space Technology, 2014. 


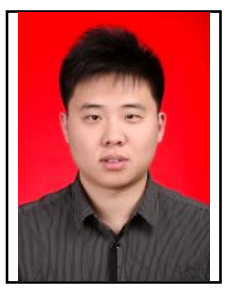

Wei Zhang is a Ph.D. candidate of China University of Petroleum in China, research direction: Enhanced geothermal system; Hydraulic fracturing; development and utilization of geothermal energy. Published literatures: Zhan-qing Qu, Wei Zhang, Tian-kui Guo. Influence of different fracture morphology on heat mining performance of enhanced geothermal systems based on COMSOL[J]. International Journal of Hydrogen Energy, 2017,42: 18263-18278. Research on the effect of geothermal reservoir parameters and bedding fractures on geothermal deliverability based on COMSOL[J]. Progress in Geophysics, 2017, 32(6):2374-2382. (in Chinese)

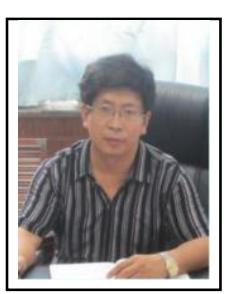

Zhan-qing Qu obtained hisPh.D. from China University of Petroleum in China, research direction: Theory and technology of oil production engineering; Hydraulic fracturing; development and utilization of geothermal energy. He is currently a professor with the China University of Petroleum. Published literatures: Zhan-qing Qu, Wei Zhang, Tian-kui Guo. Influence of different fracture morphology on heat mining performance of enhanced geothermal systems based on COMSOL[J]. International Journal of Hydrogen Energy, 2017,42: 18263-18278. D.G. Gong, Z.Q. Qu, T.K. Guo, Y. Tian, K.H. Tian. Variation rules of fracture initiation pressure and fracture starting point of hydraulic fracture in radial well[J]. Journal of Petroleum Science and Engineering, 2016, 140:41-56.

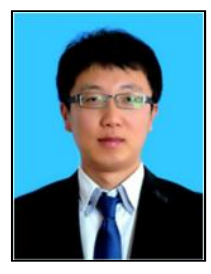

Tian-kui Guo obtained Ph.D. from China University of Petroleum in China, research direction: Hydraulic fracturing; development and utilization of geothermal energy. He is currently an associate professor with the China University of Petroleum. Published literatures: Tiankui Guo, Shicheng Zhang, Jun Gao, Jingchen Zhang, Haiqing Yu. Experimental Study of Fracture Permeability for Stimulated Reservoir Volume (SRV) in Shale Formation. Transport in porous media, 98(3):525-542, 2013. Tiankui Guo, Shicheng Zhang, Zhanqing Qu, Tong Zhou, Yongshun Xiao, Jun Gao. Experimental Study of Hydraulic Fracturing for Shale by Stimulated Reservoir Volume. Fuel, 128:373-380, 2014. Tiankui Guo, Shicheng Zhang, Hongkui Ge, Xiaoqiong Wang, Xin Lei, Bo Xiao. A New Method for Evaluation of Fracture Network Formation Capacity of Rock. Fuel, 140:778-787, 2015. 